\title{
Full Characterisation of a Focussed Extreme Ultraviolet Beam Using a Non-Redundant Array of Apertures
}

\author{
A.D. Parsons' ${ }^{1}$, P. Baksh¹, R.T. Chapman²,3 B. Mills¹, J. G. Frey², W.S. Brocklesby ${ }^{1}$ \\ 1. Optoelectronics Research Centre, University of Southampton SO17 1BJ, UK \\ 2. School of Chemistry, University of Southampton, SO17 1BJ, UK \\ 3. Rutherford Appleton Laboratories, STFC, Didcot, Oxfordshire
}

Determination of the beam parameters of a probe beam is essential for many applications across science. Full characterisation of a coherent beam requires numerous measurements of the beam profile both inside and outside the Rayleigh range to determine values of $\mathrm{w}_{0}$, the beam waist size, and $\mathrm{M}^{2}$. In the EUV (extreme ultraviolet, $\lambda<50 \mathrm{~nm})$ and X-ray $(\lambda<10 \mathrm{~nm})$ regimes, this is particularly difficult because CCD sensor pixels are too large for direct measurement and knife-edge techniques are slow due to low flux or flatness criteria and hence are subject to mechanical drifts over the exposure time. Other conventional wave-front sensing techniques such as ShackHartmann [1] sensors are limited either by high absorption in the case of lenses, or by mirror substrate flatness. Hence, a reliable, short exposure technique for beam profiling in these spectral regimes is critical for future cross-field applications at synchrotrons, XFELs (X-ray Free Electron Lasers), and lab-based sources of high energy radiation for future microscopy techniques.

We present a novel technique for characterising wavefront curvature and $\mathrm{M}^{2}$, by utilising a non-redundant array (NRA) of apertures to define the plane of investigation through an experimental EUV focus. Appropriately sampled, far-field EUV scattering from this NRA is captured on a CCD as the NRA is scanned along the beam axis through the focus. By taking the inverse Fourier transform (IFT), it is possible obtain the spatial autocorrelation functions, via the Wiener-Khinchin theorem, of the exit wave field as shown in figure 1(a). By observing the position of the first-order peaks in the autocorrelation as a function of grid translation (fig 1(b)), both the real and imaginary parts of the complex beam parameter can be determined and the $\mathrm{M}^{2}$ calculated, yielding full characterisation of the embedded Gaussian [2]. Since the periodicity of the grid is known, the planar pixel resolution can be calculated, also allowing the translations movement to be confirmed due to the change in angular acceptance of the fixed CCD. This makes the technique self-calibrating.

We demonstrate the application of this technique experimentally using a lab-based, high harmonic, EUV source at $27 \mathrm{~nm}$. The results (fig 1(c)), allow measurement of the position of the circle of least confusion [3] of a beam condensed using an off-axis Mo/Si multilayer optic accurate to $100 \mu \mathrm{m}$, and determination of the spot size $\mathrm{w}_{0}=$ $9 \mu \mathrm{m}$, and $\mathrm{M}^{2}=3$. We also investigate the limitations of this technique, by experimentally probing the impact of an increased bandwidth ( $20 \%)$, in-plane angular array misalignment and reduction of the off-axis focussing angle. The results are verified by comparison to simulations.
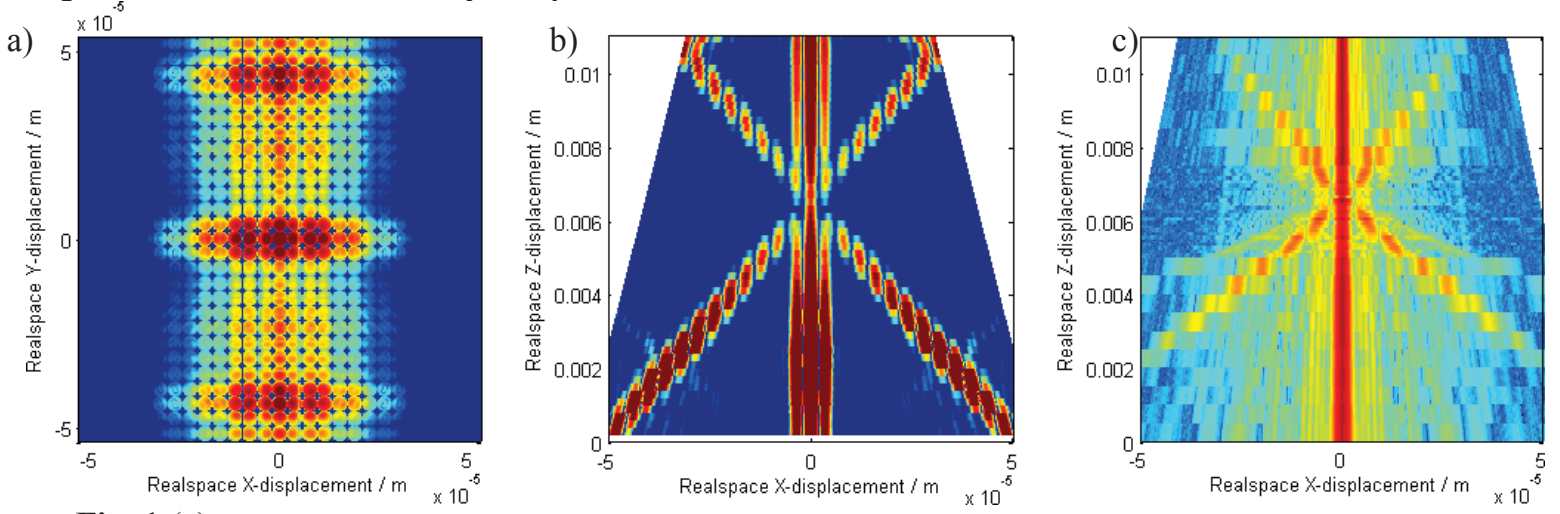

Fig. 1 (a) The 2-D autocorrelation function obtained from a simulated array of 2 micron holes illuminated by a $27 \mathrm{~nm}$ beam of EUV with a curved wavefront. First order lobe positions relative to the central maxima are related to the radius of curvature;the central maximum width to $\mathrm{w}_{0} / \mathrm{M}$. Central cross sections through the autocorrelation functions for (b) a simulated beam, and (c) for the experimental EUV data.

To conclude, we demonstrate a high impact, easy to use, cross field technique for full profiling of the embedded Gaussian of probe beams using a non-redundant array of apertures. The technique is experimentally verified in the highly absorbing EUV spectral regime, and is expected to play a significant role in other regimes, where experimental issues prevent the use of existing techniques.

\section{References}

[1]J. Hartmann et al, "Bemerkungen über den Bau und die Justirung von Spektrographen",Zt. Instrumentenkd. 29 (47) (1900)

[2]O. Svelto , "Principles of Lasers" 5th. ed. (Springer, New York, 2010, 4).

[3]B. Mills et al , "EUV off axis focussing using a High Harmonic Source," Proc. SPIE Vol. 7360736003 (2009) 